\title{
THE BACTERIOLOGY OF THE BLOOD IN EARLY LIFE *
}

FRANK SPOONER CHURCHILL, M.D., and CHARLES P. CLARK, M.D. CHICAGO

Medical literature is full of reports of the bacteriologic examinations of the blood in adults, and much valuable information has been obtained therefrom. Various infections have been studied in this way: typhoid, pneumonia, endocarditis, erysipelas, osteomyelitis, and many of the obscure febrile cases so frequently seen in our hospital wards. But while much work of this nature has been done among adults, we find comparatively few reports on this subject among infants and children. In scarlet fever alone have bacteriologic examinations of the blood been made to any extent.

Study of this infection by Jochmann, ${ }^{1}$ Baginsky, Slavyk, ${ }^{2}$ Hektoen ${ }^{3}$ and others, has shown the presence of a streptococcus, apparently identical in its characteristics with the streptococcus erysipelatis. Thus, Jochmann, studying 161 cases of scarlet fever, found the streptococcus twenty-five times. All of these patients died, and Jochmann, from analysis of his own cases and those of other authors, comes to the conclusion that the presence of this organism in the blood in scarlet fever gives an almost fatal prognosis. Curiously enough, however, neither Jochmann nor Slavyk found the streptococcus in the foudroyant cases, also invariably fatal.

Aside from these studies on scarlet fever, reports on other infections in children are meager. Rotch and Low ${ }^{4}$ report a series of thirty-six cases comprising a variety of infections, the greatest number of any single infection being typhoid (eight). They point out the scientific and practical value of taking blood cultures and urge a more widespread use of this method of investigation.

Delestre $^{5}$ examined the blood in forty cases, the patients ranging in age from a few days up to 4 years, all suffering from infections likely to prove fatal. Thirty-two of the forty died; eight recovered. Among the eight recoveries there was but one infant showing an organism in the blood, and this organism was the influenza bacillus. In the fatal cases, the blood obtained either during life or immediately after death, showed

*From the Children's Memorial Hospital.

* Read at the meeting of the American Pediatric Society, Washington, May, 1910.

1. Jochmann: Deutsch. Arch. f. klin. Med. 1903, lxxviii, 209. Ztschr. f. klin. Med. 1904, liv, 408.

2. Slavyk: Jahrb. f. Kinderh., liii, No. 5.

3. Hektoen: Jour. A. M. A., 1903, xl, 685.

4. Rotch and Low: Jour. Am. Med. Assn., 1907, xlviii, 185.

5. Delestre: Ann. d. gynec. et obst., 1901, lv, 51. 
organisms in twenty-two, in order of frequency as follows: streptococcus, staphylococcus, pneumococcus and colon bacillus.

Delestre also examined the blood from twenty-one premature infants; of these nineteen died, and fifteen of the fatal cases showed organisms in the blood, the order of frequency being as follows: streptococcus, colon bacillus, staphylococcus and pneumococcus. The author attributes this susceptibility of the newly-born to bacteremia, to the extensive absorbing surface in the lungs and intestines, and regards these tracts as the main points of entry of the bacteria, though the umbilicus, of course, is another vulnerable point. He gives few details in his cases, not stating either the clinical or anatomic diagnosis. The blood taken during life was obtained by an incision into the skin, and he believes his technic was so nearly aseptic that there was no danger of contamination.

Otten $^{6}$ reports a series of 250 cases of lobar pneumonia in children up to 15 years of age, with blood-cultures in seventy. He found the pneumococcus in nine of these seventy cases, 13 per cent.; of these nine, four patients recovered; five died.

The paucity of studies on this important subject in children is undoubtedly due to the difficulty of obtaining sufficient blood for examination from this class of patients. The veins of children are so small that it is extremely difficult to get into them. We have ourselves experienced this difficulty, and, besides the cases reported below, have made numerous but unsuccessful attempts to obtain the blood from other patients, chiefly infants and young children. Others apparently have had the same experience. Indeed, one author, studying a large series of infections of otitic origin among adults, "throws up the sponge" at the outset and says that no attempt is made to study children on account of the difficulty of carrying out the technic. But considering the frequency of obscure febrile infections among infants and children, it would seem that in such patients especially, systematic bacteriologic blood-examinations might be of great value; furthermore, in view of the future possibilities of antivaccine and antiserum treatment, accurate knowledge of disease is more than ever desirable; hence any method of investigation which promises to give us this accurate knowledge should be thoroughly tested, and, if prolific of results, systematically employed. It seems worth while, therefore, to report the following cases, even though small in number:

\section{TECHNIC}

Most of the cases have been studied at the Children's Memorial Hospital, a few each at the Cook County and Presbyterian hospitals, and to the attending physicians at these institutions we are much indebted for the material used.

6. Otten: Jahrb. f. Kinderh., 1909, Ixix, 568. 
The blood has been obtained by the usual method, from the median basilic vein just above the elbow. In two cases of suspected typhoid it was obtained from the lobe of the ear, as recommended by Peabody. Various kinds of syringes have been used, but the Luer glass-syringe has been the most satisfactory. The blood thus obtained has been mixed immediately at the bedside with various media: in most of the cases two tubes of bouillon, two of agar and agar plate have been inoculated. In five of the typhoid cases, ox-bile has been used as the medium, with positive results in all. A case of suspected typhoid in which bile medium was used was negative, but proved later to be a case of tuberculous meningitis.

In using the ox-bile as a medium, the following technic was observed:

Test tubes containing fresh ox-gall sterilized in the autoclave were used. The blood obtained from the vein, or from the lobe of the ear in some cases, was introduced directly into the test-tube of bile. This was then put in the incubator from fifteen to eighteen hours, at the end of which time a few loopfuls were transferred to a tube of coagulated bloodserum: after three to five hours of incubation microscopic examination of the water of condensation showed, in the positive cases, the presence of motile bacilli. The presence of such motile bacilli is now accepted as evidence of a typhoid or a paratyphoid organism.

We have made sixty-nine examinations on sixty-four infants and children during life, and have studied sixteen cases post mortem. It is with the former group of cases that we are chiefly concerned. This is composed of the following infections:

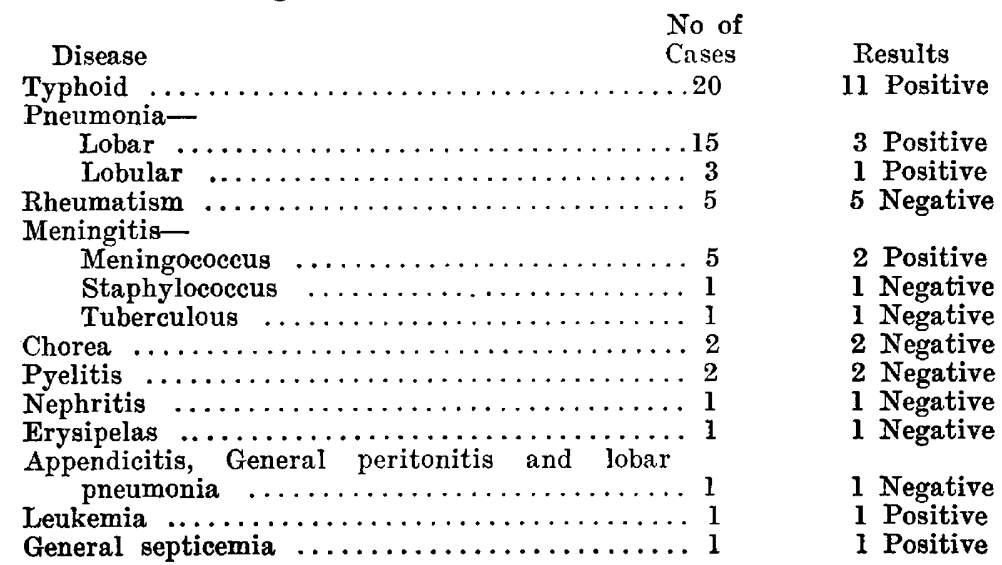

In addition to the above one case each of scorbutus, miliary tuberculosis, arthritis deformans, toxic erythema and of tuberculous adenitis, gave negative results. Thus, of the sixty-nine examinations made on these sixty-four children, we have obtained nineteen positive results. These will now be analyzed according to individual infections. 


\section{TYPHOID}

It is in this infection perhaps that the greatest amount of blood-culture work has been done among adults, with interesting and important results. During the first week of the disease, 95 to 100 per cent. of the patients will show the presence of typhoid bacilli in the circulating blood: with the progress of the disease, this number diminishes so that in the second or third week they are found much less frequently (40 per cent.). As the Widal reaction pursues exactly the reverse course, absent at first, present later, the blood culture test becomes an extremely important method in the early diagnosis of typhoid, at least among adults.

How is it among children? Our own cases are of course too few to warrant conclusions. We can state only that eleven of the twenty cases gave positive results, and that these positive results were obtained as early as the sixth and as late as the twenty-second day (Table 1). The negative results were obtained in the first case on the tenth, thirteenth and twentieth days, in the second case during the seventh week (relapse), and in the third and fourth cases on the eighth day.

Combining Rotch's series, our own and a few scattering reports in literature, we find thirty-one cases of typhoid fever, in twenty, or 66 per cent. of which, bacilli were found in the circulating blood. We find, furthermore, that they were found as early as four days before the onset of symptoms and as late as the twenty-second day. The former case was that of a 12-year-old child whose sister was already ill with typhoid (Conradi). ${ }^{7}$

These results were obtained with all kinds of media and at all periods of the disease. It is probable that with the more widespread use of Conradi's ox-bile medium and more frequent examinations early in the disease, this proportion of positive results will be considerably increased. Only when large numbers of cases have been examined, and at different periods of the disease, can we draw final conclusions as to the importance of this particular laboratory investigation. If the results of the future correspond with the results already obtained in large numbers of adults, this method will become even more valuable among children on account of the more irregular, atypical nature of the disease in early life. We urge a constant, widespread use of the method, that our knowledge in this direction may be increased as rapidly as possible.

\section{PNEOMONIA}

The pneumococcus is a frequent invader of the blood in adults, not only in pneumonia, but also in pneumococcic infections with the pathologic seat of the disease in other localities, e. g., the endocardium and pericardium, the joints, the meninges, the tonsils, etc. As in typhoid the literature is voluminous as to adults, meager as to children.

7. Conradi: Deutsch. med. Wchnschr., 1906, xxxii, 58. 
Otten studied 250 cases of pneumonia in children up to 15 years of age. He made blood-cultures on seventy of these patients, finding the pneumococcus in nine, or 13 per cent. Of these nine children four recovered, five died.

Spitta $^{8}$ describes a case of lobar pneumonia in a 17 months' infant, with a general pneumococcic infection, resulting in an arthritis and empyema; pneumococci were found in the blood during the sixth week of illness and one week before death. Autopsy confirmed the antemortem examinations.

We have taken cultures in fifteen cases of lobar pneumonia and three of lobular pneumonia. Four of the cases in the former group were positive, one in the latter, the organism found being the pneumococcus in every instance.

Thus in the eighty-five cases comprising our own and Otten's series, thirteen, or 15 per cent., showed pneumococci in the circulating blood, a striking difference from its frequency among similar infections in adults. This failure to discover the organism more frequently in the blood in children is due to one of the two causes. Either the technic employed in the cases already studied is faulty, or the organism does not invade the blood frequently. The technic employed is apparently important, great discrepancies existing among the authors who have studied this question in adults. Thus Lenhartz, Schottmüller, Grimm and Jochmann find pneumococei in the blood in only one-fourth to two-thirds of their cases, whereas Procharska and Weins find them in practically all of their cases. The former group of observers worked with solid media-bloodagar; the latter with fluid media--blood bouillon.

To this difference in culture media, Otten attributes the varying results obtained by these workers, maintaining that the pneumococcus grows better on fluid media, and that consequently those who use such media will get a comparatively high percentage of positive results. $\mathrm{He}$ himself in his work on children used fluid media, yet got positive results in only 13 per cent. of his cases. Consequently, if his reasoning be correct, the pneumococcus is not a frequent invader of the blood in the lobar pneumonia of children. However, here again the cases available for study are too few to warrant final conclusions, either as to the frequency of its appearance or as to its prognostic value when present. Future investigations must determine these points.

\section{THE RHEUMATIC AFFECTIONS}

In this group we have included cases of endocarditis, arthritis, tonsillitis and chorea, these manifestations occurring either singly or in combination with each other (Table 1 ).

8. Spitta: Brit. Med. Jour., 1902, ii, 1579. 


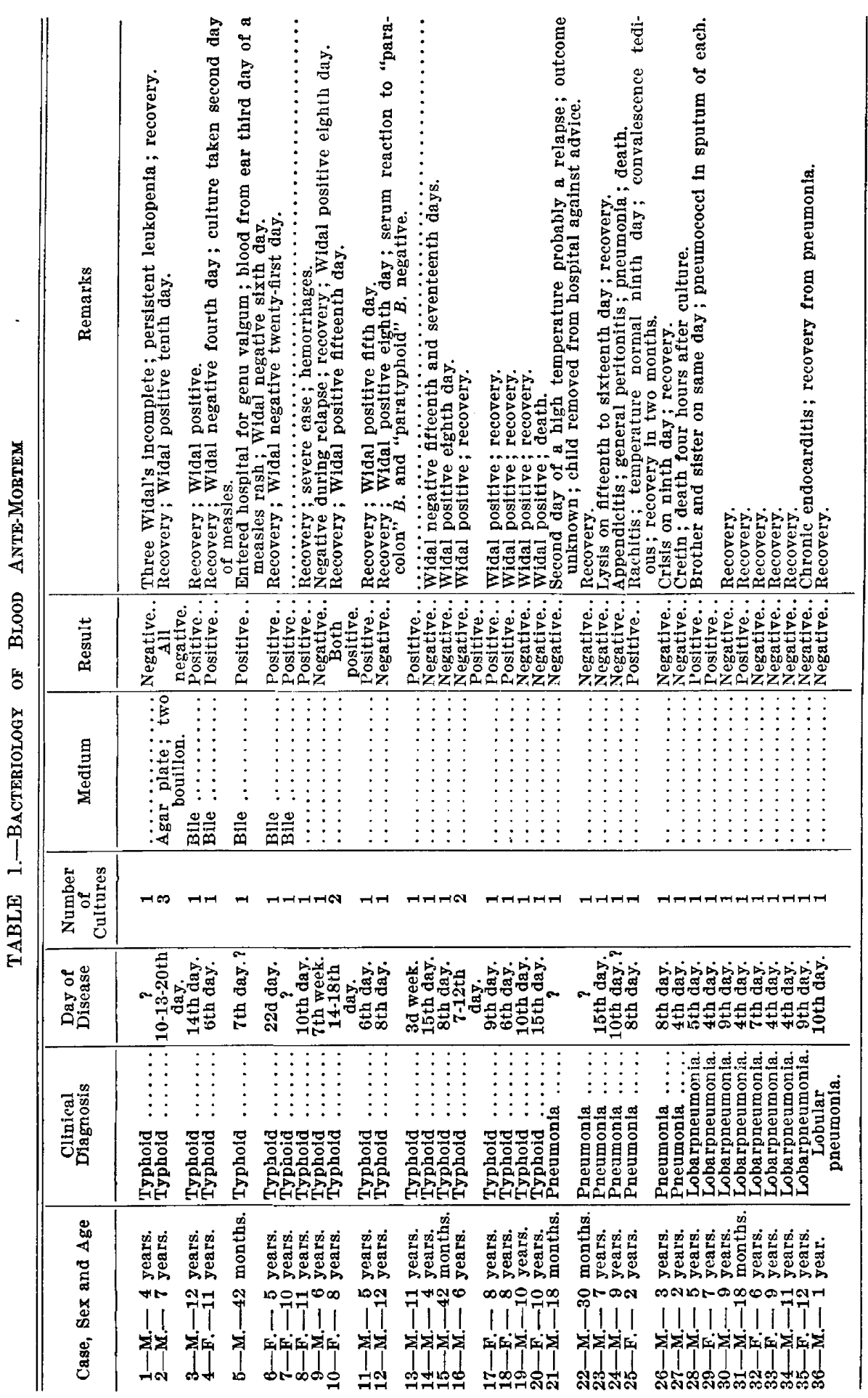




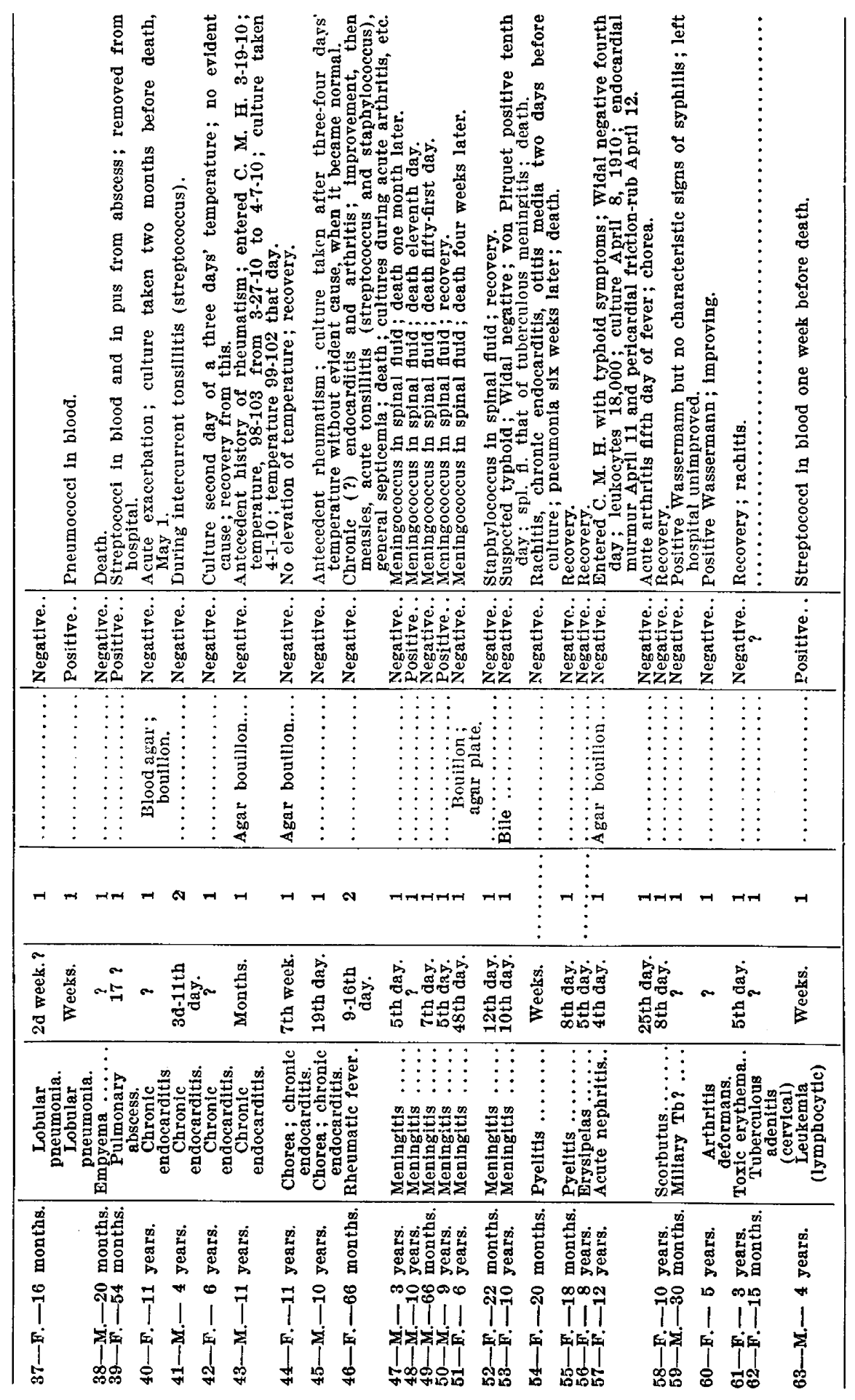


Post-mortem examinations in cases of endocarditis have shown a varied bacteriology, streptococci, staphylococci, pneumococci, gonococci, pyocyaneus and influenza bacillus, all having been found in the heart's blood and on the valves of the heart in such patients. During life, in cases of septic endocarditis in adults, bacteria have often been found in the blood (Billings). We know practically nothing of this aspect of the subject in children, however. Our own group comprises seven cases; all had chronic endocarditis; in five of the cases, the cultures were taken during a febrile stage, due either to a tonsillitis, an arthritis, or to an unknown cause, possibly an acute exacerbation of the chronic endocarditis. There were two cases of chorea, and in both of these the temperature was normal when the cultures were taken. Cultures were negative in all seven of the cases.

\section{MENINGITIS}

It was formerly thought that in epidemic meningitis the meningococcus rarely invaded the blood, but recent investigations with improved technic show its presence in the blood in about 25 per cent. of the cases. We have examined four cases, with two positive results. In the tuberculous case the patient was examined on the tenth day of the disease as a suspected typhoid.

\section{OTHER DISEASES}

One case each of pyelitis, erysipelas and acute nephritis gave negative blood-cultures. Catheterized specimens of urine from the cases of pyelitis and nephritis showed a streptococcus in the former and nothing in the latter. One case of acute lymphocytic leukemia showed the streptococcus in the blood one week before death.

\section{POST MORTEM}

Much more work has been done on the bacteriology of the blood on children post mortem than ante mortem. After death the blood often shows the presence of organisms in great variety. We have reports on the bacteriology of the heart's blood in seventeen cases, of which thirteen showed positive results. These cases comprised the usual run of hospital patients: intestinal, pulmonary, bronchial; otitic, etc. (Table 2 ). Three cases of special interest were, one each of hemorrhagic disease of the newly born, erysipelas and status lymphaticus. The hemorrhagic disease showed in the heart's blood the Staphylococcus aureus, the bacillus coli, the streptococcus and an unidentified diplococcus midway between the pneumococcus and the streptococcus.

Of the four cases with a sterile heart's blood, three were as follows: A pyelo-nephritis due to the $B$. pyocyaneus, an otitis with a general septicemia, but with entirely negative post-mortem results, and a meningo- 


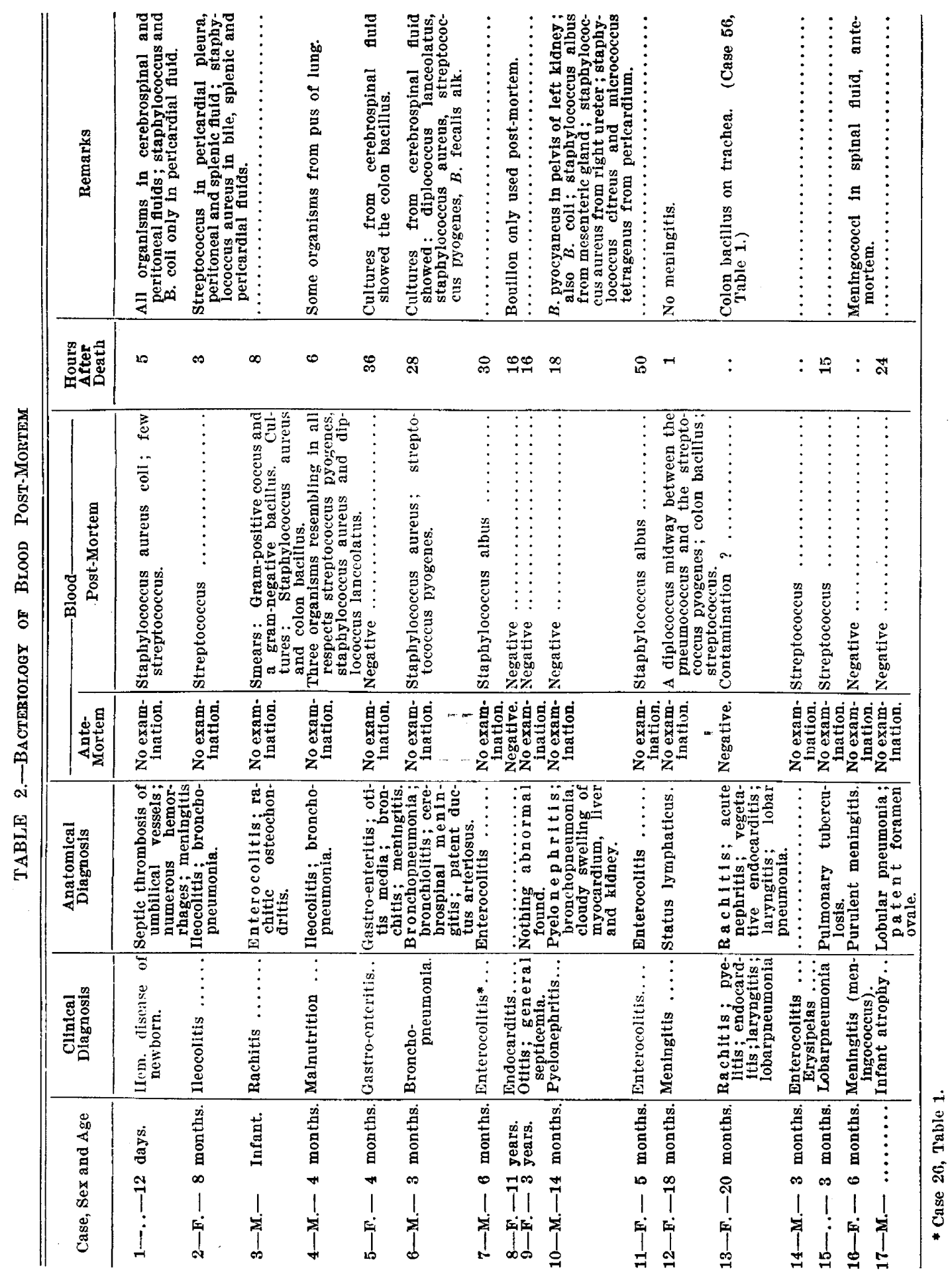


coccic meningitis. The fourth case was an endocarditis in which the antemortem blood was also sterile (Case 26, Table 2).

The organisms recovered post mortem were as follows: the staphylococcus six times, aureus five times, albus one time, the streptococcus eight times, bacillus coli three times, the Diplococcus lanceolatus one time, and the unidentified diplococcus already mentioned.

We are much indebted to Prof. E. R. LeCount of the Pathological Department of Rush Medical College, under whose supervision most of the post-mortem work in our cases was done.

\section{SUMMARY}

The main points in this paper may be briefly summarized as follows: Blood-cultures in adults during life, in large numbers of cases, have yielded information of scientific interest and diagnostic value.

Little work in this direction has been done in the infections of early life. There is need of more of this work among infants and children.

It is reasonable to suppose that blood-cultures in early life may be of even more value than in adult life, on account of the greater frequency of obscure infections during the former period.

1259 North State Street. 